Panorama Económico, Volumen V, No. 9, julio-diciembre, 2009, pp. 137-159

\title{
EVALUACIÓN DE LA POLÍTICA DE LA COMPETENCIA EN ALEMANIA
}

\author{
Sergio A. Berumen* \\ Octavio A. Palacios Sommer**
}

\section{RESUMEN}

En el pasado las autoridades responsables del gobierno alemán se han limitado a reaccionar ante las presiones que ha ejercido la Comisión Europea en materia de la competencia pero, salvo muy escasas excepciones, los distintos gobiernos de este país han actuado de forma reactiva. En este artículo se muestra que la política de la competencia de Alemania ha sido errática: en ocasiones la autoridad ha actuado oportunamente pero ha habido otras en las que resultaba urgente su intervención y ésta se ha hecho esperar. Para el efecto, el interés se centra en analizar la evolución del mercado alemán y en señalar los avances más significativos que se han alcanzado en materia de liberalización del mercado interior entre 2004 y 2008.

Palabras clave: Economía alemana, política de la competencia Clasificación JEL: L13, L41, L43

\begin{abstract}
In the past the responsible authorities of the German government have limited their performance to react to those pressures coming from the European

* Profesor titular. Universidad Rey Juan Carlos. Subdirector de Libros de Economía y Empresa (Real Academia de Ciencias Morales y Políticas). Correo electrónico: <lee_berumen@ racmyp.es>. ** Profesor asociado. Escuela Superior de Economía, del Instituto Politécnico Nacional.
\end{abstract}


Commission in respect to competence matters, but only in some exceptional circumstances the different German governments have acted in a reactive way. In this article it is demonstrated that the German policies regarding competence have been erratic; sometimes the authorities have reacted in time, but in some other occasions their intervention was quite urgent and it has arrived later than it was expected. To this effect, interest is focused in analyzing the evolution of the German market and in pointing out the most significative advances that have been reached in respect to their internal market liberalization, between 2004 and 2008.

Keywords: German economy, competition policy

Jel classification: L13, L41, L43

\section{INTRODUCCIÓN}

Para Glos (2007) la garantía y protección de la competencia es una de las tareas fundamentales y centrales del gobierno. A su parecer, el actual gobierno alemán está interesado en "proteger" y "asegurar" la competencia en todos los sectores. Sin embargo, en sus declaraciones se ha omitido el fomento de la misma, lo que da a pensar que el gobierno está interesado en proteger lo ya conseguido pero no en plantearse nuevas metas. Lamentablemente esta actitud no es nueva.

En el pasado la economía alemana se ha limitado a reaccionar ante las presiones que han ejercido los burócratas competentes desde la Comisión Europea (particularmente de Neelie Kroes, actual Comisaria de la Competencia) pero, salvo contadas excepciones, los distintos gobiernos han actuado de forma reactiva. Así se ha puesto de manifiesto en el caso de la abolición de los monopolios del sector energético o del correo postal: en ambos casos se produjo la liberalización sólo después de intensas presiones ejercidas desde Bruselas. No obstante, también hay una cara más positiva de la política de la competencia alemana en materia de lucha contra la piratería y el contrabando, entre lo que destaca el acuerdo firmado con el gobierno chino. Lo anterior revela que la política de la competencia alemana ha sido errática. En ocasio- 
nes la autoridad ha actuado oportunamente, pero ha habido otras en las que resultaba urgente su intervención y ésta se ha hecho esperar. En este artículo veremos si el actual gobierno alemán ha cumplido con las expectativas señaladas por Glos o si sólo se ha tratado de buenas intenciones, pero que no han conducido a hechos concretos. Para el efecto, nos daremos a la tarea de analizar la evolución del mercado alemán y señalaremos los avances más significativos que se han alcanzado en materia de liberalización del mercado interior entre 2004 y 2008.

\section{LIBERALIZACIÓN DEL MERCADO INTERIOR}

\subsection{DESREGULACIÓN}

El primer paso para identificar la posición de Alemania en materia de política de la competencia es consultar el Index of Economic Freedom (Índice de Libertad Económica) que publica la Heritage Foundation (2008). Este país ocupa el vigésimo tercer lugar en el ranking mundial (entre 157 países analizados en el 2008)

y el duodécimo en el europeo en materia de libertad económica, de lo que se desprende que, en principio, aún queda un largo camino por recorrer hasta alcanzar a los países punteros, como Irlanda y Suiza.

El segundo documento que permite identificar los esfuerzos realizados en materia de desregulación es el Economic Freedom of the World Report (Informe sobre la libertad económica en el mundo) que publica el Instituto Fraser (2008). Según este documento Alemania ocupaba el puesto décimo octavo entre 141 países analizados en el 2005. Mientras que el Index of Economic Freedom estima que el mercado alemán se puede considerar "libre" en un 71,2\%, el Economic Freedom of the World Report lo sitúa en un 76\%. A primera vista la evaluación obtenida parece satisfactoria, en especial si consideramos que el porcentaje está por encima del promedio mundial. Pero cabe destacar que el puntaje general en el 2008 fue 0,4\% más bajo que el del año anterior. Es decir que, en vez de progresar en materia de desregulación este país ha sufrido un retroceso. 
Los dos informes antes citados coinciden en que existe un alto grado de libertad en los sectores: i) comercial; ii) del comercio exterior; iii) de los derechos de propiedad industrial e intelectual; y iv) en todo lo referente a la inversión y el crédito. Pero hace falta mejorar en materia de: i) libertad fiscal; ii) en el tamaño del sector público; y iii) en lo referente a flexibilidad laboral.

En lo concerniente al tamaño del sector público, el actual gobierno de la Canciller Angela Merkel, entre 2006 y 2008 ha realizado denodados esfuerzos para reducir el tamaño del aparato burocrático, pero el margen de maniobra de que dispone es francamente estrecho debido a que en este país prevalece un formidable Estado de Bienestar y le precede una larga historia de intervencionismo estatal en prácticamente todas las áreas de la economía. A modo de ejemplo, en la Tabla 1 se aprecia el elevado gasto que ha ejercido el gobierno en el Sector Privado, a través de generosas ayudas y subvenciones entre 2003 y 2005.

\section{TABLA 1}

Gasto del gobierno alemán por sectores

\begin{tabular}{|c|c|c|c|c|c|r|}
\hline \multicolumn{1}{|c|}{ Alemania } & \multicolumn{2}{|c|}{$\mathbf{2 0 0 3}$} & \multicolumn{2}{c|}{$\mathbf{2 0 0 4}$} & \multicolumn{2}{c|}{$\mathbf{2 0 0 5}$} \\
Rankings & \multicolumn{2}{|c|}{$7,4(19)$} & \multicolumn{2}{c|}{$7,6(17)$} & \multicolumn{2}{c|}{$7,6(18)$} \\
\hline Tamaño del gobierno & 4,4 & & 5,5 & & 5,7 & \\
A. Gasto del gobierno orientado al consumo & 4,5 & $(24,8)$ & 4,7 & $(23,9)$ & 4,7 & $(24,1)$ \\
B. Transferencias y subsidios & 2,0 & $(29,8)$ & 2,3 & $(28,6)$ & 2,3 & $(28,8)$ \\
C. Empresas e inversiones gubernamentales & 6,0 & & 10,0 & $(9,2)$ & 10,0 & $(9,3)$ \\
D. Tasa marginal por concepto de impuestos: & 5,0 & & 5,0 & & 6,0 & \\
i) Tasa marginal de ingresos por & 5,0 & $(47)$ & 5,0 & $(46)$ & 6,0 & $(44)$ \\
$\quad \begin{array}{l}\text { impuestos } \\
\text { Tasa marginal de ingresos por } \\
\text { nóminas }\end{array}$ & 5,0 & $(47)$ & 5,0 & $(46)$ & 6,0 & (44) \\
& & & & & & \\
\hline
\end{tabular}

Fuente: Fraser Institute, Economic Freedom of the World Report, Vancouver B.C, Fraser Institute, 2008. 
Una muestra de lo anterior fue que entre 2000 y 2003, en tiempos del gobierno de Gerhard Schröder del Sozialdemokratische Partei Deutschlands (SPD-Partido Social Demócrata Alemán), este país incumplió el Pacto de Estabilidad y Crecimiento señalado en el seno de la Unión Económica y Monetaria (UEM) de la Unión Europea (UE) al contraer una deuda superior al 3\% del PIB acordado (sobre este particular véase el trabajo de Sinn, 2008). En las elecciones de mayo de 2005 el Christlich Demokratische Union Deutschlands (CDU-Partido Democristiano Alemán) de Angela Merkel obtuvo la mayoría de los votos, lo que le permitió conformar la alianza de partidos de diferente color que gobernará hasta 2009. En esta alianza, sin embargo, el SPD tiene una presencia poderosa, lo que se ha traducido en una fuerte reducción en la implementación de las reformas que son más necesarias para enfrentar los desafíos estructurales de la economía. Desde el principio de la legislatura (22 de noviembre de 2005) el SPD se ha esforzado en oponerse en todo aquello que esté relacionado con la desregulación, fundamentalmente en materia de salarios mínimos.

Los gastos del Sector Público alemán alcanzan el 47\% del PIB. Esta exagerada cifra se conforma principalmente por los altos subsidios de desempleo. Por esta razón, en la denominada Agenda 2010 (Agenda zwanzig-zehn; originalmente ésta fue planteada por Schröder en marzo de 2003, pero posteriormente fue adoptada por Angela Merkel en noviembre de 2005) la actual Canciller planteó un programa orientado a reestructurar las bases del Estado de Bienestar, en especial en materia sanitaria y del mercado laboral. La propuesta definitiva se centraba en la reducción del Arbeitslosengeld (subsidio por desempleo) y de la Arbeitslosenhilfe (prestación por desempleo), con lo cual se pretendía incentivar a los trabajadores para que se empleasen por su cuenta y, de ese modo, reducir la tasa de desempleo y los consecuentes costos que le representa al Estado. La llamada Ich-AG era una ayuda financiera para todos aquellos que deseasen crear su propia empresa. Sin embargo, y pese a todas las bondades que presentaba la Agenda 2010, el proyecto fracasó. Los inmensos vacíos legales a los que se enfrentó ha significado que el Estado ha tenido que seguir soportando la inmensa carga derivada del viejo esquema social. El prin- 
cipal detractor de la Agenda 2010 fue el Freien Demokratischen Partei (FDP-Partido Liberal Democrático, 2005), bajo el argumento de que los costos sociales y parafiscales serían demasiado elevados. La parte más negativa de todo este cúmulo de decisiones ha sido que las empresas no han tenido los suficientes incentivos para emplear a más trabajadores, si bien existe, paradójicamente, una demanda real de mano de obra.

Como señala Harms (2007), la economía alemana es de las más poderosas del mundo, pero la carga que representa el sostenimiento del actual Estado de Bienestar es excesivamente oneroso. Sobre este particular, es evidente que el proceso de desregulación se ha hecho mejor y más rápido en el Reino Unido, los Países Bajos e Irlanda, que en Alemania e Italia.

\subsection{DESARME ARANCELARIO}

En Alemania las tasas aduaneras se refieren a contribuciones e impuestos que se originan en el intercambio comercial con terceros países, es decir, con países no miembros de la UE. Por tanto, la percepción de aranceles está relacionada con el intercambio del comercio (de bienes y servicios) en el seno de la UE, dado que al interior del territorio Comunitario las barreras arancelarias se han desmantelado por completo. La cuantía de los aranceles a pagar se ajusta al Arancel Aduanero Común (AAC). El AAC se circunscribió en el marco de la Unión Aduanera en 1968, en el cual los países miembros acordaron abolir sus tarifas nacionales e introducir una tarifa única comunitaria frente a terceros países. A partir de la creación del Mercado Único Europeo (en 1993) también se eliminaron los controles de aduana entre los países miembros. De acuerdo al criterio adoptado por el Ministerio de Economía y Tecnología (2008 (1)), en la actualidad todo lo relacionado a cuestiones de derecho arancelario se sustenta en el Código Aduanero Comunitario de 1994, en las Disposiciones de Aplicación que le acompañan y en el AAC.

Adicionalmente, la legislación alemana se complementa con la Comunitaria en los casos del llamado Einfuhrumsatzsteuer (Impuesto a la importación), el cual es un impuesto adicional que grava la importación de bienes proceden- 
tes de terceros países (es semejante al impuesto sobre ventas que se aplica en los países miembros de la UE). Esto significa que, el Estado alemán impone un impuesto a los bienes importados de países extracomunitarios con el interés de impedir que éstos lleguen al consumidor final sin haber sido gravados con impuestos indirectos. A pesar de que el Einfuhrumsatzsteuer es una tasa únicamente alemana, está sujeta a las disposiciones señaladas en la legislación Comunitaria de 1993. La segunda excepción al AAC son las "suspensiones" y los “contingentes autónomos", señalados en el artículo 26 del Tratado de Roma (BF, 2008). Durante un determinado período de tiempo se permite la exención total o parcial de los derechos aduaneros que se suelen aplicar a las importaciones de materias primas y bienes semielaborados, siempre que no estén disponibles en cantidades suficientes en la UE o en terceros países que gocen de derechos preferenciales. En realidad se trata de suspensiones o de contingentes autónomos en función de las cantidades (EC, 2008(1)).

En la actualidad las autoridades alemanas han priorizado las discusiones que se están llevando a cabo en Bruselas en materia de fomento de la competencia. En tiempos recientes la Comisión Europea ha mostrado singular interés en publicar un libro blanco que contenga un elenco de las posibles reformas legislativas en el ámbito del derecho de indemnización por violación de la Gesetz gegen Wettbewerbsbeschränkungen (GWB-Ley de la competencia, del 27 de julio de 1957), en las cuales se destaque todo lo referente a la protección de los derechos de los consumidores. Por su parte, el gobierno alemán se ha dado a la tarea de adaptar la legislación interna a los posibles cambios en la legislación Comunitaria. Los más críticos a la labor de Angela Merkel han denunciado el papel pasivo que ha asumido el gobierno en el fomento de la competencia, dado que en realidad sólo ha actuado a instancias de las presiones que recibe desde las instituciones Comunitarias, pero no motu-proprio.

En lo referente a la relación que subyace entre la GWB alemana y la legislación Comunitaria, como no puede ser de otro modo, prevalecen las directivas de las UE, si bien hay ciertas excepciones. A modo de ejemplo, en el campo de la prohibición de cárteles, el artículo 81 del Tratado de la CEE y la GWB se pueden aplicar paralelamente: mientras que la imposición del artículo 
81 es obligatoria, la de la ley nacional alemana es facultativa. Asimismo, se aplican los artículos 19 a 21 del GWB en cuanto a la inspección de abusos en la imposición de precios, dado que los reglamentos nacionales son más estrictos que los Comunitarios.

\subsection{FOMENTO DE LA COMPETENCIA EN EL MERCADO INTERIOR}

El Ministerio de Economía y Tecnología (BWT, 2008 (2)) alemán reconoce que los objetivos de la política económica se sustentan en el mantenimiento de los mercados en pleno funcionamiento. Considera que la existencia de un mercado competitivo es una condición imprescindible para el crecimiento y el empleo, dado que aporta una serie de ventajas que impactan positivamente en el ámbito de la generación de innovaciones, en la mejor distribución de recursos y en el cumplimiento de los derechos de los consumidores. Los tres principios señalados en la GWB (2008) son: i) luchar contra los cárteles y en general contra cualquier tipo de acciones que restrinjan la competencia; ii) señalar los lineamientos específicos en materia de fusiones y adquisiciones; y iii) garantizar la transparencia en la redacción y el cumplimiento de los contratos entre el Estado y los sectores productivos privados.

En el 2005 la GWB fue modificada con el interés de ajustarla al Derecho Comunitario de la Competencia, en concreto con lo señalado en el Reglamento 1/2003 de la UE. Adicionalmente, el 26 de septiembre de 2006 la BundesKartellamt (BK-Oficina Federal de Cárteles) publicó nuevas pautas para la determinación de multas en caso de violación de la GWB, y dos años más tarde (en diciembre de 2007) se añadieron nuevas disposiciones contra el abuso de precios en los sectores de suministro de energía y en el de los alimentos (OECD, 2006). Los párrafos añadidos penalizan el incremento desorbitado de los precios del gas y la venta de alimentos por debajo de su costo de producción (dumping).

En materia alimentaria se prohíbe el dumping salvo justificación objetiva, como podría ser la de evitar que caduquen los alimentos. Los beneficiarios 
directos de las modificaciones han sido las pequeñas y medianas empresas porque éstas han sido las que históricamente se han visto más perjudicadas por la competencia de precios, lo que no deja de ser cuestionable porque, después de todo, en realidad las nuevas disposiciones son una deliberada intervención en el mercado alimentario, como así lo ha denunciado la Organización para la Cooperación y el Desarrollo Económico (OCDE, 2007). Por el contrario, el argumento planteado por la BK señala que la revisión de la legislación en materia de energía se debió principalmente a la insuficiente competencia que prevalecía y a los constantes e injustificados incrementos en los precios que tenían lugar en este mercado. En realidad, gracias a los cambios promovidos por la BK ahora existen mejores herramientas jurídicas para luchar contra el incremento de precios exorbitantes en el suministro de gas y electricidad. Una muestra de ello son los litigios que la BK ha entablado contra 35 distribuidores de gas (hasta mayo de 2008) (véase Vorwurf, 2008).

Como consecuencia a la modificación de la Ley, los proveedores ahora están obligados a desvelar sus cómputos internos para demostrar que el aumento de las tarifas es justificable (anteriormente la carga probatoria reposaba en la BK). Sin embargo, y si bien la modificación a la Ley ha sido un paso importante en el proceso de liberalización, el mercado del suministro de energía está lejos de ser todo lo competitivo que debería, como lo han reconocido las propias autoridades de la BK.

La BK es la responsable de la imposición de las sanciones en materia de competencia. Su sede central está en Bonn, pero en todo el país hay oficinas regionales para todo lo que afecte a los respectivos Länder (estados federados). Esta Oficina es la encargada de controlar las fusiones entre empresas cuya facturación conjunta no supere los cinco billones de euros, debido a que a partir de este límite es competencia de la Comisión Europea (véase Beck, 2006). Su trabajo se especializa en supervisar las concentraciones de agentes en el mercado que puedan vulnerar los principios del libre mercado, tales como la conformación de monopolios y oligopolios.

Desde marzo de 2002 la BK dispone de una comisión especial denominada Sonderkommission Kartellbekämpfung (SKK-Comisión Especial de lucha 
contra los Monopolios), cuya misión es la de observar el funcionamiento de la competencia en los sectores económicos de este país. Entre sus funciones destacan: i) evaluar las tendencias en materia de concentración de mercados; ii) identificar la formación de cárteles en materia de acuerdos sobre precios; y iii) en lo referente a la fijación de cuotas de venta. Para el efecto, la SKK regularmente presenta informes a la BK y al gobierno federal para que tengan conocimiento del estado de las cosas.

Asimismo, la SKK es el punto de contacto para las empresas que desean recurrir a la Bonusregelung (Reglamentación de bono). Ésta es una cláusula (fue introducida en 2000 y sometida a revisión y actualización en marzo de 2006) que concede un acuerdo a las empresas que están implicadas en prácticas ilegales, y que les permite evitar parcial o incluso totalmente las multas si contribuyen notablemente a la revelación de las prácticas que atentan contra la competencia de parte de otras empresas. Tal y como lo ha señalado la BK (2007 (1)), esta reglamentación ha resultado ser muy eficaz, como lo muestran las 76 solicitudes que se presentaron en el 2006 y que se referían a 19 casos distintos. En la Tabla 2 se puede apreciar la evolución de los índices que miden la eficacia de las autoridades responsables en la materia. Estos datos revelan que en los últimos años los sectores productivos más sancionados han sido los de cemento y hormigón, y papel.

En lo referente al control de concentraciones de empresas, en el 2006 se inscribieron 1,829 fusiones. Este número es superior al antiguo record de 1,735 notificaciones de 2000, lo que refleja el dinamismo que está registrando la economía de este país. De los 1,829 proyectos de fusión presentados, la BK sólo impidió 5, lo que equivale al 0,27\% (Deutscher Bundestag, 2007). En la Tabla 3 se aprecia la evolución de las fusiones inscritas y la relación de las concentraciones impedidas entre 2000 y 2006. Resulta llamativo que el número de notificaciones ha sido relativamente constante en los últimos años mientras que el número de prohibiciones no presenta ninguna tendencia evidente (si bien en ningún caso supera el 1\%). A la luz de los datos se puede concluir que el Estado alemán interviene más bien poco en materia de fusiones entre empresas, lo que significa que es partidario de permitir la autoregulación del mercado. 
TABla 2

Evolución de las sanciones

(2001-2006)

\begin{tabular}{|c|c|c|c|c|c|c|}
\hline & 2001 & 2002 & 2003 & 2004 & 2005 & 2006 \\
\hline $\begin{array}{l}\text { Importe multas } \\
\text { (en millones de } € \text { ) }\end{array}$ & 21,8 & 4,5 & 717,0 & 58,0 & 163,9 & 4,5 \\
\hline $\begin{array}{l}\mathrm{N}^{\circ} \text { de registros a } \\
\text { nivel nacional }\end{array}$ & -- & 8 & 7 & 11 & 6 & 4 \\
\hline Casos más relevantes & $\begin{array}{l}\text { Sectores d } \\
\text { hormiq } \\
\text { producci }\end{array}$ & $\begin{array}{l}\text { ento y } \\
\text { de } \\
\text { apel }\end{array}$ & $\begin{array}{r}\text { Sectores d } \\
\text { horm } \\
\text { produccic }\end{array}$ & $\begin{array}{l}\text { ento y } \\
\text { apel }\end{array}$ & \multicolumn{2}{|c|}{$\begin{array}{l}\text { Aseguradoras, agencias } \\
\text { de transportes, sectores de } \\
\text { cemento y hormigón }\end{array}$} \\
\hline
\end{tabular}

Fuente: Bundeskartellamt, Bundeskartellamt legt Tätigkeitsbericht 2001/2006, Bonn, Bundeskartellamt, 2007 (2), pp. 16-23.

TABLA 3

Evolución de las fusiones y su relación de concentraciones sancionadas (2000-2006)

\begin{tabular}{crrrrrrr}
\hline & $\mathbf{2 0 0 0}$ & $\mathbf{2 0 0 1}$ & $\mathbf{2 0 0 2}$ & $\mathbf{2 0 0 3}$ & $\mathbf{2 0 0 4}$ & $\mathbf{2 0 0 5}$ & $\mathbf{2 0 0 6}$ \\
$\mathrm{n}^{\circ}$ notificaciones & 1735 & 1568 & 1584 & 1366 & 1412 & 1687 & 1829 \\
$\mathrm{n}^{\circ}$ prohibiciones & 2 & 2 & 6 & 2 & 12 & 6 & 5 \\
$\%$ prohibiciones & $0,12 \%$ & $0,13 \%$ & $0,38 \%$ & $0,15 \%$ & $0,85 \%$ & $0,36 \%$ & $0,27 \%$ \\
\hline
\end{tabular}

Fuente: Bundeskartellamt, Bundeskartellamt legt Tätigkeitsbericht 2001/2006, Bonn, Bundeskartellamt, 2007 (2), pp. 21-25. 
El trabajo realizado por la BK es muy relevante, pese a que no siempre ha contado con el suficiente apoyo del gobierno federal. Una muestra de ello es que en el 2007 a esta Oficina se le dotó con un presupuesto de 17 millones de euros, lo que significó un aumento de 0,3 millones de euros en relación al del año anterior, equivalente al $2 \%$. Si consideramos que la tasa de inflación en ese año fue del $2,2 \%$, es evidente que este incremento fue escasamente significativo (Bundeskartellamt, 2008).

\section{AVANCES EN MATERIA DE LUCHA CONTRA LOS MONOPOLIOS Y OLIGOPOLIOS}

La lucha contra el monopolio del correo postal es un ejemplo muy actual de los avances alcanzados en la materia. Mientras que en el mercado del envío de paquetes ha habido una intensa competencia desde hace años; hasta finales de 2007 la Deutsche Post AG (DP) gozó de una situación de monopolio en el envío de correo postal. Entre 1989 y 1995 la DP fue privatizada, y desde noviembre de 2000 cotizan en Bolsa el 30\% de sus acciones; sin embargo, diversos bancos estatales, como el Kreditinstitut für Wiederaufbau (KfWInstituto de crédito para la reconstrucción), siguen detentando un peso importante en el porcentaje de acciones y en las decisiones que asume el grupo (Bundesnetzagentur, 2007).

En diversas ocasiones los competidores han acusado a DP de ejercer prácticas monopolísticas, como lo prueba su negación en 2005 de permitir que las empresas de la competencia tuviesen acceso a los canales de distribución del correo postal. Después de haber examinado el caso, tanto la BK como la Comisión Europea concluyeron que se trataba de un impedimento a la competencia y ordenaron que el resto de las empresas pudiesen acceder al sistema de distribucin postal. La negativa de DP se sustentaba en la licencia exclusiva para el reparto de correo postal que el Estado le había concedido, y que implicaba que un determinado porcentaje del mercado estuviera reservado para ella. El proceso de apertura inici el 11 de febrero de 2005, pero no fue sino 
hasta el 1 de enero de 2008 cuando qued definitivamente abolido el monopolio del que gozaba la antigua empresa estatal. Como se puede intuir, desde que se estipul el final definitivo del monopolio ejercido por DP ha habido una evolución dinámica en el mercado postal. En la actualidad hay aproximadamente 750 empresas que compiten en este sector productivo, si bien sólo dos operan en todo el territorio alemán. En la Tabla 4 se aprecia el comportamiento que registr este mercado entre 2001 y 2007.

\section{TABLA 4}

Evolución de las cuotas de mercado de competidores y DP (2001-2007)

\begin{tabular}{|c|c|c|c|c|c|c|c|}
\hline & 2001 & 2002 & 2003 & 2004 & 2005 & 2006 & 2007 \\
\hline $\begin{array}{l}\text { Mercado en total (en } \\
\text { millones de } € \text { ) }\end{array}$ & 10237 & 10164 & 9900 & 9994 & 9863 & 10073 & 10060 \\
\hline $\begin{array}{l}\text { Facturación de } \\
\text { competidores } \\
\text { (en millones de } € \text { ) }\end{array}$ & 249 & 305 & 388 & 532 & 745 & 1056 & 1274 \\
\hline $\begin{array}{l}\text { Cuota de mercado } \\
\text { competidores }\end{array}$ & $2,4 \%$ & $3,0 \%$ & $3,9 \%$ & $5,3 \%$ & $7,6 \%$ & $10,5 \%$ & $12,7 \%$ \\
\hline $\begin{array}{l}\text { Cuota de mercado de } \\
\text { DP }\end{array}$ & $97,6 \%$ & $97,0 \%$ & $96,1 \%$ & $94,7 \%$ & $92,4 \%$ & $89,5 \%$ & $87,3 \%$ \\
\hline
\end{tabular}

Fuente: Bundesnetzagentur, Tätigkeitsbericht 2006/2007 der Bundesnetzagentur für Elektrizität, Gas, Telekomunikation, Post und Eisenbahenen, Bonn, Bundersnetzagentur, 2007, pp. 18 y sig.

Estas cifras revelan que si bien ha habido una notable apertura del sector postal alemán aún no se puede hablar de competencia plena. Muestra de ello es que en 2007 el conjunto de empresas competidoras reunieron el 12,7\% del volumen total de negocios, mientras que la DP concentró el 87,3\% del mismo. Estos resultados se deben a que DP se beneficia del libre acceso que tiene a la infraestructura estatal y a que ha sabido mantener su mercado natural. Empero, la cuestión más controvertida es que DP sigue gozando de notables e injustificadas ventajas en materia fiscal. A modo de ejemplo, Storn (2004) señala que esta empresa está exenta del impuesto sobre el tráfico de empresas, lo que le proporciona una considerable ventaja frente a sus compe- 
tidores que están obligados a pagar el total del impuesto. Son este tipo de acciones las que incitan a pensar que la Administración Pública alemana realiza un doble juego. Por una parte pretende abolir el monopolio en el sector, pero por otro lado se asegura que DP tenga una buena posición de partida en un mercado competitivo.

El segundo caso que exponemos es el de la distribución de energía. En 1998 el sector fue liberalizado debido a dos directivas Comunitarias (la primera de 1996 y la segunda de 1998), pero lo cierto es que aún está lejos de consumarse una genuina libre competencia en el ramo. Las cuatro más grandes empresas suministradoras de energía (E.ON, RWE, Vattenfall y EnBW) producen el $80 \%$ de la corriente y poseen la totalidad de la red eléctrica (OECD, 2007(2)). A cambio de una tasa, estas empresas están obligadas a poner a disposición de los demás proveedores (que son demasiado pequeños para construir su propio sistema de suministro) las redes eléctricas. Consecuentemente, las empresas dominantes gozan de un monopolio natural, gracias a lo cual no se ven enfrentadas a una fuerte competencia.

En la práctica las cuatro más grandes empresas se han repartido el mercado por regiones territoriales, lo que las convierte en un cártel. Tal proceder ha despertado el recelo de las autoridades de la BK y de la Comisión Europea, las cuales han emprendido acciones legales (la primera contra $R W E$ en mayo de 2006 y la segunda en julio del mismo año contra E.ON). Con la intención de que la Comisión sobreseyera los procedimientos, en febrero de 2008 E.ON ofreció vender su parte de la red eléctrica alemana a terceros. La propuesta se acogió con satisfacción en Bruselas, pero en Berlín provocó indignación, dado que el gobierno de Angela Merkel en varias ocasiones se había manifestado en contra de la enajenación (EC, 2008(2)). Este caso particular demuestra que los operadores de este país le conceden mayor importancia a las decisiones de la Unión Europea que a las de su gobierno federal.

Desde julio de 2005 la Bundesnetzagentur (2008) (Agencia Federal de Red) es la responsable de supervisar el sector energético, específicamente en materia de tarifas. La labor de esta Agencia se centra en: i) aprobar las tasas; ii) impedir o eliminar barreras de entrada al mercado; iii) estandarizar los pro- 
cesos para el cambio de suministradores; y iv) gestionar la mejora de la conexión entre las centrales eléctricas a la red ya existente. Su decisión más relevante hasta la fecha ha sido que en enero de 2008 esta Agencia obligó a los cuatro operadores a bajar las tasas para la utilización de sus redes por terceras empresas.

\section{AVANCES EN MATERIA DE LUCHA CONTRA LA PIRATERÍA Y EL CONTRABANDO}

La diferencia fundamental entre contrabando y piratería consiste en las características de los bienes que se comercian: mientras que el contrabando es el tráfico ilegal de productos legales, los piratas trafican artículos imitados ilegalmente. La autoridad encargada de cuantificar el daño generado en ambas materias es la Verband Deutscher Maschinen und Anlagenbau (VDMAAsociación de Construcción de Maquinaria Alemana). Algunos de los datos más relevantes de los últimos cinco años señalan que los sectores productivos de este país se han visto afectados en 4,5 billones de euros al año, lo que se ha traducido en la destrucción de 700,000 puestos de trabajo y en que el $80 \%$ de las empresas se han visto impactadas (Bascap, 2008).

Según la Zollbehörde (Autoridad aduanera), que es la responsable de combatir la piratería y el contrabando, el número de productos confiscados en el 2006 aumentó en más de 550\% en relación al año anterior. Tal incremento ha obedecido a una mayor eficiencia y eficacia en materia de cooperación policial y judicial entre todos los países miembros de la UE. A partir del 1 de julio de 2004 se ha intensificado la labor gracias a la directiva comunitaria VOEG$1383 / 2003$, en virtud de la cual las agencias tributarias y arancelarias están facultadas para combatir sendos delitos en toda la órbita Comunitaria, y ya no sólo en el ámbito de las fronteras nacionales de los países miembros. Naturalmente, la medida ha sido muy eficaz.

La Tabla 5 muestra que en la mayoría de los casos los productos confiscados han sido falsificaciones (es decir, piratería), mientras que el contrabando 
de mercancía no imitada (como cigarrillos y alimentos) sólo ha constituido un porcentaje insignificante. En especial es relevante la reducción en la incautación de cigarrillos a partir de 2004 (ver recuadro interno); la explicación es que, en mayo de ese año se ampliaron las fronteras de la UE hacia el Este, de modo que en adelante se les permitió a los consumidores alemanes importar legalmente los cigarrillos que provenían de países del Este. Es decir que, este mercado pasó de ser ilegal a ser legal.

\section{TABLA 5}

\section{Reparto de los productos por categorías y porcentaje de las confiscaciones}

\begin{tabular}{lccrr}
\multicolumn{4}{c}{$(\mathbf{2 0 0 3}-\mathbf{2 0 0 6})$} & \\
\hline & $\mathbf{2 0 0 3}$ & $\mathbf{2 0 0 4}$ & $\mathbf{2 0 0 5}$ & $\mathbf{2 0 0 6}$ \\
Ropa de deporte & 16,53 & 30,18 & 7,21 & 7,97 \\
Accesorios (bolsos etc.) & 20,48 & 21,21 & 20,22 & 18,45 \\
Ropa de ocio & 23,29 & 19,69 & 24,20 & 25,67 \\
Relojes y Joyería & 14,82 & 11,14 & 20,75 & 18,09 \\
Otros (piezas de & 7,13 & 4,85 & 9,55 & 7,26 \\
recambio, etiquetas etc.) & & & & \\
Electrodomésticos & 1,59 & 4,38 & 8,32 & 8,20 \\
Discos y Software & 6,01 & 2,81 & 2,60 & 4,65 \\
Juguetes & 5,09 & 2,45 & 2,68 & 2,39 \\
\hline Cigarrillos & 3,50 & 2,02 & 0,24 & 0,35 \\
Perfumes y Cosmética & 0,78 & 0,79 & 3,47 & 4,79 \\
Ordenadores & 0,61 & 0,32 & 0,57 & 0,58 \\
Alimentos & 0,17 & 0,16 & 0,19 & 0,12 \\
Medicamentos & & & & 1,48 \\
\hline
\end{tabular}

Fuente: Bascap, Produktpirateria in Deutschland, Berlin, Business Action to Stop Counterfeiting and Piracy, 2008.

En materia de legislación sobre ambos delitos, el Estado alemán cuenta con la Gesetz über Urheberrecht und verwandte Schutzrechte (Ley del Derecho del Autor, del 9 de septiembre de 1965, y reformada en octubre de 2006), en la cual se señalan medidas más severas contra los fabricantes, traficantes y vendedores de piratería y, en concreto, en lo referente a las descargas ilegales de 
música y películas de Internet. La experiencia derivada del éxito en cuestiones de piratería y contrabando en parte se ha fundado en los diversos acuerdos que ha firmado el gobierno con algunos de los países exportadores de los productos piratas. Como se muestra en la Tabla 6, China es el país de origen de la mayor parte de los artículos imitados. Por ello, en junio de 2000 el gobierno del canciller Gerhard Schröder promovió la firma del tratado Deutsch-Chinesische Vereinbarung zu dem Austausch und der Zusammenarbeit im Rechtsbereich (Tratado chino-alemán sobre el intercambio y la cooperación en cuestiones de derechos). Este documento sentó las bases para intensificar las relaciones bilaterales en la materia entre los dos países y para la formación de grupos de negociación para reducir el contrabando y la piratería proveniente del país asiático (BJ, 1999).

\section{Tabla 6}

Porcentajes de las confiscaciones de acuerdo al país de origen

\begin{tabular}{|c|c|c|c|c|}
\hline \multicolumn{5}{|c|}{$(2003-2006)$} \\
\hline & 2003 & 2004 & 2005 & 2006 \\
\hline China & 12,86 & 23,58 & 35,80 & 32,85 \\
\hline Tailandia & 24,85 & 23,45 & 10,20 & 8,61 \\
\hline Turquía & 9,04 & 10,22 & 8,73 & 8,71 \\
\hline Estados Unidos & 3,81 & 8,44 & 11,23 & 12,66 \\
\hline Hong-Kong & 5,87 & 7,68 & 11,45 & 11,12 \\
\hline República Checa & 11,44 & 3,57 & 0,97 & 0,80 \\
\hline Polonia & 10,03 & 3,56 & 0,59 & 0,56 \\
\hline India & -- & 1,79 & - & 1,36 \\
\hline Malaysia & 3,64 & 1,31 & -- & 0,58 \\
\hline Japón & -- & -- & 0,45 & 0,76 \\
\hline Emiratos Árabes & -- & -- & 1,27 & 1,63 \\
\hline \multicolumn{5}{|l|}{ Unidos } \\
\hline Vietnam & -- & -- & -- & 2,72 \\
\hline Suiza & -- & -- & -- & 1,19 \\
\hline Otros & 18,46 & 16,40 & 19,31 & 16,47 \\
\hline \multicolumn{5}{|c|}{$\begin{array}{l}\text { Otros: Afganistán, Kosovo, Canadá, Corea del Sur, Brasil, Singapur, Taiwán, Bulgaria, Rusia, } \\
\text { Ucrania, Irán, Filipinas, Pakistán, Uzbekistán, Croacia, Argentina, España, Israel, Eslovaquia } \\
\text { y Australia. }\end{array}$} \\
\hline
\end{tabular}

Fuente: Bascap, Produktpirateria in Deutschland, Berlin, Business Action to Stop Counterfeiting and Piracy, 2008. 
Los resultados derivados de la firma de este Tratado, sin embargo, son insuficientes. Como muestra: un tercio de los productos confiscados por las autoridades alemanas fue fabricado en China (como se puede apreciar en la tabla anterior). Angela Merkel, actual canciller, ha puesto en duda el éxito del Tratado y ha criticado la insuficiente protección de propiedad intelectual de las autoridades chinas, en especial en lo referente a la piratería de recambios automotrices (véase IAA, 2007).

\section{EVALUACIÓN DE LOS PROCESOS DE PRIVATIZACIÓN MÁS REPRESENTATIVOS}

La Bundeshaushaltsordnung (Ley de contabilidad, del 19 de agosto de 1969) es la legislación especializada en lo referente a la regulación de los procesos de privatización (BJ, 1969). Para el efecto, ésta señala que antes de privatizar una empresa pública es obligatorio la creación de grupos de trabajo que estén conformados por expertos del gobierno, cuya tarea será la de comprobar que la operación esperada no contraviene las disposiciones legales y que la ejecución de los servicios básicos está garantizada con posterioridad a la venta. Tras la caída del Muro de Berlín y la consiguiente unificación del país en 1989, tuvo lugar una ola de privatizaciones de empresas de la extinta República Democrática de Alemania (RDA), donde el $88 \%$ de las empresas eran de carácter estatal, pero en los últimos años no ha habido especial actividad en estos temas, probablemente por que quedan muy pocas empresas que sean susceptibles de ser privatizadas (BF, 2007).

Dos de las privatizaciones más emblemáticas en los últimos años han sido las de Deutsche Telekom AG y la de Fraport AG. Deutsche Telekom AG (Telekom) fue el resultado de la transformación obligatoria de la antigua Deutsche Bundespost por la Postreform (Ley para la reforma de Correos, de enero de 1995). La operación implicó que la Deutsche Bundespost se dividiese en tres empresas independientes, todas ellas serían sociedades anónimas. Bajo el mandato de Ron Sommer, presidente de la Junta Directiva, desde el 18 
de noviembre de 1996 las acciones de Telekom cotizan en Bolsa. En la primera emisión de acciones el Estado vendió un cuarto de sus participaciones, por lo cual ingresó 10 billones de euros; posteriormente le siguieron dos emisiones (en 1999 y 2000), en las cuales el Estado percibió otros 23 billones de euros. Como consecuencia de la privatización, en la actualidad el Estado alemán detenta el $31,7 \%$ de las acciones (del cual el $17 \%$ pertenecen al $\mathrm{KfW}$ ), otro $62,8 \%$ se encuentran en libre flotación y el restante $4,5 \%$ está en manos de la empresa estadounidense Blackstone (BF, 2008).

En la actualidad, Telekom es la empresa de telecomunicaciones más grande de Europa; emplea a más de 240,000 trabajadores y en el 2008 facturó un volumen de negocios superior a los 62,5 billones de euros. René Obermann (2007), actual Presidente de la Junta Directiva, reconoce que en los próximos años su empresa tendrá que enfrentarse a una competencia cada vez más agresiva y diversa, para lo cual ha decidido apostar muy fuertemente por los procesos de innovación.

La privatización de Fraport AG tuvo lugar en 2001. Esta empresa gestiona el aeropuerto de Frankfurt (Frankfurt International Airport y RheinMain-Flughafen), considerado como el octavo aeropuerto más transitado del mundo, detenta el 30\% del aeropuerto de Hannover y el 65\% del aeropuerto Frankfurt Hahn. También es la propietaria de los aeropuertos de Anatolia (Turquía), El Cairo (Egipto), Lima (Perú) y Nueva Delhi (India). Desde su inauguración en 1947 hasta 2001 (año en que empezó a cotizar en Bolsa), el propietario del aeropuerto de Frankfurt era el Estado (el municipio de Frankfurt poseía el $29 \%$, el Länd de Bundesland Hessen, el 45\% y el Estado federal el 26\%).

En 2005, el Estado federal vendió sus participaciones por 660 millones de euros a varias empresas, entre las que destacan Lufthansa AG y Julis Bär Holding, con el 10 y el 5\% respectivamente. En la actualidad el $25 \%$ de las acciones está en libre flotación (incluyendo las participaciones que están en manos de los empleados), mientras que las participaciones del municipio de Frankfurt y las de Bundesland Hessen constituyen el 20 y el 32,5\% respectivamente (Reuters, 2005). Como se puede apreciar, la mayoría de las acciones de esta empresa continúan en manos de entes públicos. 
La privatización de Fraport $A G$ no se puede considerar exitosa debido a que en la actualidad el Estado sigue siendo el propietario mayoritario. Por el contrario, la privatización Deutsche Telekom ha sido moderadamente exitosa. Aparte de esto, hay que destacar que el objetivo de la exaltada "política de reformas" no parece ser la reducción del papel del Estado y el fomento de la competencia, sino más bien la reducción de la Deuda Pública. Las recientes privatizaciones de varios hospitales también encajan en este contexto: fueron los municipios los que llevaron a cabo estas operaciones con el interés de compensar el déficit en sus balances.

\section{CONCLUSIONES}

En el pasado la economía alemana ha reaccionado ante las presiones que ha ejercido la Comisión Europea en materia de la competencia, pero salvo contadas excepciones los distintos gobiernos han actuado de forma reactiva. Como lo señalan el Index of Economic Freedom y el Economic Freedom of the World Report, este país tiene intensos retos que enfrentar en materia de desregulación. Uno de los mayores desafíos del actual gobierno de Angela Merkel es el de la difícil tarea de implementar los principios originalmente planteados en la Agenda 2010, especialmente en lo referente a la redefinición de las bases del Estado de Bienestar, en concreto con lo relacionado a: i) intervencionismo estatal en la economía; ii) reducción de subsidios de desempleo; iii) en materia sanitaria; y iv) del mercado laboral. Para el efecto, el punto de partida consiste en reducir el subsidio por desempleo y la prestación por desempleo, con lo cual los trabajadores tendrán mayores estímulos para emplearse por su cuenta.

En términos generales el tejido jurídico e institucional con los que cuenta la economía de Alemania en materia de la competencia es bastante avanzado. Una muestra de ello es que cuenta con sólidas herramientas jurídicas para luchar contra el incremento de precios exorbitantes y la formación de cárteles. Posiblemente el elemento más importante contra este tipo de prácticas es la 
denominada reglamentación de bono, la cual es un acuerdo que se pone a disposición de las empresas que están implicadas en prácticas ilegales, y que les permite evitar parcial o incluso totalmente las multas si contribuyen a la revelación de las prácticas que atentan contra la competencia de parte de otras empresas.

De la información aquí expuesta se desprende que es necesario redoblar esfuerzos en materia de lucha contra los monopolios. Una muestra de ello es que en 2007 el conjunto de empresas especializadas en el envío de paquetes conformaba el $12,7 \%$ del volumen total de negocios, mientras que la Deutsche Post $A G$ concentraba el 87,3\% del mismo. En el caso de la distribución de energía, las cuatro más grandes empresas suministradoras (E.ON, RWE, Vattenfall y $E n B W$ ) producen el $80 \%$ de la corriente y poseen la totalidad de la red eléctrica. La cuestión es que entre éstas se han repartido el mercado por regiones territoriales, lo que las convierte en un cártel, algo incompatible con los principios que defiende la comisaria Neelie Kroes.

Finalmente, en materia de privatizaciones los avances logrados son ambivalentes. Por una parte se han privatizado prácticamente todas las empresas que representaban algún atractivo para el sector privado pero, por otra parte, aún existen casos en los que hay un largo camino por recorrer. Los dos ejemplos más emblemáticos son los de Telekom y el de Fraport AG. En la primera el Estado alemán detenta el 31,7\% de las acciones, mientras que en la segunda el municipio de Frankfurt posee el $20 \%$ y el Länd de Bundesland Hessen el 32,5\%. Sendos porcentajes denotan que el Estado aún tiene una presencia demasiado potente en ámbitos donde su participación resulta cada día más cuestionable.

\section{BIBLIOGRAFÍA}

Bascap, Produktpirateria in Deutschland, Berlin, Business Action to Stop Counterfeiting and Piracy, 2008.

Beck, M., Bayer darf Schering schlucken, Berlin, Focus, 2006. 
BF, Privatisierung von Bundesunternehmen (Unmittelbare Beteiligungsunternehmen des Bundes), Berlin, Bundesministerium der Finanzen, 2008.

BF, Rechtsgrundlagen für die Erhebung von Zöllen, Berlin, Bundesministerium der Finanzen, 2008.

BJ, Bundeshaushaltsordnung (BHO), Bonn, Bundesministerium der Justiz, 1969.

BJ, Deutsch-Chinesische Vereinbarung zu dem Austausch und der Zusammenarbeit im Rechtsbereich, Berlin, Bundesministerium der Justiz, 1999.

Bundeskartellamt, Bericht des Bundeskartellamtes über seine Tätigkeit in den Jahren sowie über die Lage und Entwicklung auf seinem Aufgabengebiet, Bonn, Bundeskartellamt, 2007(1).

Bundeskartellamt, Bundeskartellamt legt Tätigkeitsbericht 2001-2006, Bonn, Bundeskartellamt, 2007(2).

Bundeskartellamt, Über den Erlass und die Reduktion von Geldbußen in Kartellsachen, Bonn, Bundeskartellamt, 2008.

Bundesnetzagentur, Bericht zur Auswertung der Netzzustands-und Netzausbauberichte der deutschen Elektrizitätsübertragungsnetzbetreiber, Bonn, Bundesnetzagentur, 2008.

Bundesnetzagentur, Tätigkeitsbericht 2006 / 2007 der Bundesnetzagentur für Elektrizität, Gas, Telekomunikation, Post und Eisenbahnen, Bonn, Bundesnetzagentur, 2007.

BWT, Autonome Zollaussetzungen/Zollkontingente (AZZ): Instrument, Bonn, Bundesministerium für Wirtschaft und Technologie, 2008(1).

BWT, Gesetz gegen den unlauteren Wettbewerb (UWG), Bonn, Bundesministerium für Wirtschaft und Technologie, 2008(2).

Deutscher Bundestag, Wirtschaft und Technologie/Unterrichtung, Berlin, Deutscher Bundestag, 2007.

EC, Antitrust: Commission on E.ON proposals for structural remedies to increase competition in German electricity market, Brussels, European Comission, 2008(2).

EC, Calculation of Custom Duties, Brussels, European Comission, 2008(1). 
FDP, Das Präsidium der Freien Demokratischen Partei hat auf seiner Sitzung, Berlin, Freien Demokratischen Partei, 2005.

Fraser Institute, Economic Freedom of the World Report, Vancouver B.C, Fraser Institute, 2008.

Gesetz gegen Wettbewerbsbeschränkungen, Berlin, 2008.

Gesetz über Urheberrecht und verwandte Schutzrechte (Urheberrechtsgesetz), Berlin, 2008.

Glos, M., "Wettbewerb als Garant einer freiheitlichen Wirtschafts - und Gesellschaftsordnung", Internationalen Kartellkonferenz / des 14. Europäischen Wettbewerbstages, Marzo 2007.

Harms, P., Deregulation and the Current Account, Konstanz, Study Center Gerzensee and University of Konstanz, 2007.

Heritage Foundation, Index of Economic Freedom, Washington D.C., Heritage Foundation, 2008.

IAA, "Keine China - Kopien in Frankfurt", Focus, 30-08-2007.

Obermann, R., Die Deutsche Telekom AG auf einen Blick, Bonn, Telekom, 2007.

OECD, Annual Report on Competition Policy Developments in Germany, Paris, Organisation for Economic Co-operation and Development, 2006.

OECD, Annual Report on Competition Policy Developments in Germany, Paris, Organisation for Economic Co-operation and Development, 2007(1).

OECD, Annual Report on Competition Policy Developments in Germany, Paris, Organisation for Economic Co-operation and Development, 2007(2).

Reuters, "Bund wirft Fraport - Aktien auf den Markt", Der Spiegel, Müchen, 2610-2005.

Sinn, H-B, "Brandbrief der deutschen Wirtschaftsforscher", Sueddeutsche, 13/ 03/2008, München.

Storn, A., Bröckelndes Monopol, Bonn, Die Zeit, 2004.

Vorwurf, P., Kartellamt, Der Spiegel, Müchen, 2008. 